\title{
On the connection between accreting X-ray and radio millisecond pulsars
}

\author{
T. M. Tauris ${ }^{1,2, *}$ \\ ${ }^{1}$ Argelander-Institut für Astronomie, Universität Bonn, Germany \\ ${ }^{2}$ Max-Planck Institut für Radioastronomie, Bonn, Germany \\ *email: tauris@astro.uni-bonn.de
}

\begin{abstract}
For many years it has been recognized that the terminal stages of mass transfer in a low-mass X-ray binary (LMXB) should cause the magnetosphere of the accreting neutron star to expand, leading to a braking torque acting on the spinning pulsar. After the discovery of radio millisecond pulsars (MSPs) it was therefore somewhat a paradox (e.g. Ruderman et al. 1989) how these pulsars could retain their fast spins following the Roche-lobe decoupling phase, RLDP. Here I present a solution to this so-called "turn-off problem" which was recently found by combining binary stellar evolution models with torque computations (Tauris 2012). The solution is that during the RLDP the spin equilibrium of the pulsar is broken and therefore it remains a fast spinning object. I briefly discuss these findings in view of the two observed spin distributions in the populations of accreting X-ray millisecond pulsars (AXMSPs) and radio MSPs.
\end{abstract}

Keywords. stars: neutron, pulsars: general, X-rays: binaries, stars: rotation

\section{Introduction}

Theoretical modelling of accretion torques with disk-magnetosphere interactions have been performed for nearly four decades (e.g. Ghosh \& Lamb 1979). Likewise, detailed calculations of binary evolution have demonstrated that both the duration of the RLO in LMXBs and the amounts of matter transfered are adequate to fully recycle a pulsar (e.g. Tauris \& Savonije 1999; Podsiadlowski et al. 2002). However, these previous studies did not combine numerical stellar evolution calculations with computations of the resulting accretion torque at work. For further details on these issues, and more general discussions of my results, I refer to the journal paper, Tauris (2012).

\section{The magnetosphere-disk interactions}

The interplay between the neutron star magnetic field and the conducting plasma in the accretion disk is a rather complex process. The physics of the transition zone from Keplerian disk to magnetospheric flow is important and determines the angular momentum exchange from the differential rotation between the disk and the neutron star (e.g. Spruit \& Taam 1993). It is the mass transfered from the donor star which carries this angular momentum which eventually spins up the rotating neutron star once its surface magnetic flux density, $B$ is low enough to allow for efficient accretion.

The gain in neutron star spin angular momentum can approximately be expressed as: $\Delta J_{\star}=\sqrt{G M r_{\text {mag }}} \Delta M \xi$, where $\xi \simeq 1$ is a numerical factor which depends on the flow pattern (Ghosh \& Lamb 1979), $\Delta M=\dot{M} \cdot \Delta t$ is the amount of mass accreted in a time interval $\Delta t$ with average mass accretion rate $\dot{M}$ and $r_{\text {mag }}$ is the magnetospheric boundary, roughly located at the inner edge of the disk; i.e. $r_{\mathrm{mag}}=\phi \cdot r_{\mathrm{Alfven}}$, where the magnetospheric coupling parameter, $\phi=0.5-1.4$, Wang (1997); D'Angelo \& Spruit (2010). 
For the numerical calculations of the RLDP, I included the effect of additional spin-down torques, acting on the neutron star, due to both magnetic field drag on the accretion disk (Rapapport et al. 2004) as well as magnetic dipole radiation, although these effects are usually not dominant. The total spin torque can be written as:

$$
N_{\text {total }}=n(\omega)\left(\dot{M} \sqrt{G M r_{\mathrm{mag}}} \xi+\frac{\mu^{2}}{9 r_{\mathrm{mag}}^{3}}\right)-\frac{\dot{E}_{\text {dipole }}}{\Omega}
$$

where $n(\omega)=\tanh \left((1-\omega) / \delta_{\omega}\right)$ is a dimensionless function, depending on the fastness parameter, $\omega=\Omega_{\star} / \Omega_{\mathrm{K}}\left(r_{\mathrm{mag}}\right)=\left(r_{\mathrm{mag}} / r_{\mathrm{co}}\right)^{3 / 2}$, which is introduced to model a gradual torque change in a transition zone near the magnetospheric boundary.

\section{Roche-lobe decoupling phase (RLDP)}

The rapidly decreasing mass-transfer rate during the RLDP results in an expanding magnetosphere which causes a significant braking torque to act on the spinning pulsar. Thus it forces the rotational period to increase, as shown in Fig. 1. At some point the spin equilibrium is broken. Initially, the spin can remain in equilibrium by adapting to the decreasing value of $\dot{M}$. Further into the RLDP, however, $r_{\text {mag }}$ increases on a timescale faster than the spin-relaxation timescale, $t_{\text {torque }}$ at which the torque can transmit the effect of deceleration to the neutron star and therefore $r_{\mathrm{mag}}>r_{\mathrm{co}}$, leading to $P<P_{\mathrm{eq}}$ at all times onwards. The results is that AXMSPs in LMXBs typically lose $\sim 50 \%$ of their rotational energy (see Fig. 2), depending on the B-field of the pulsar, the duration of the RLDP and the assumptions governing the disk-magnetosphere interactions.

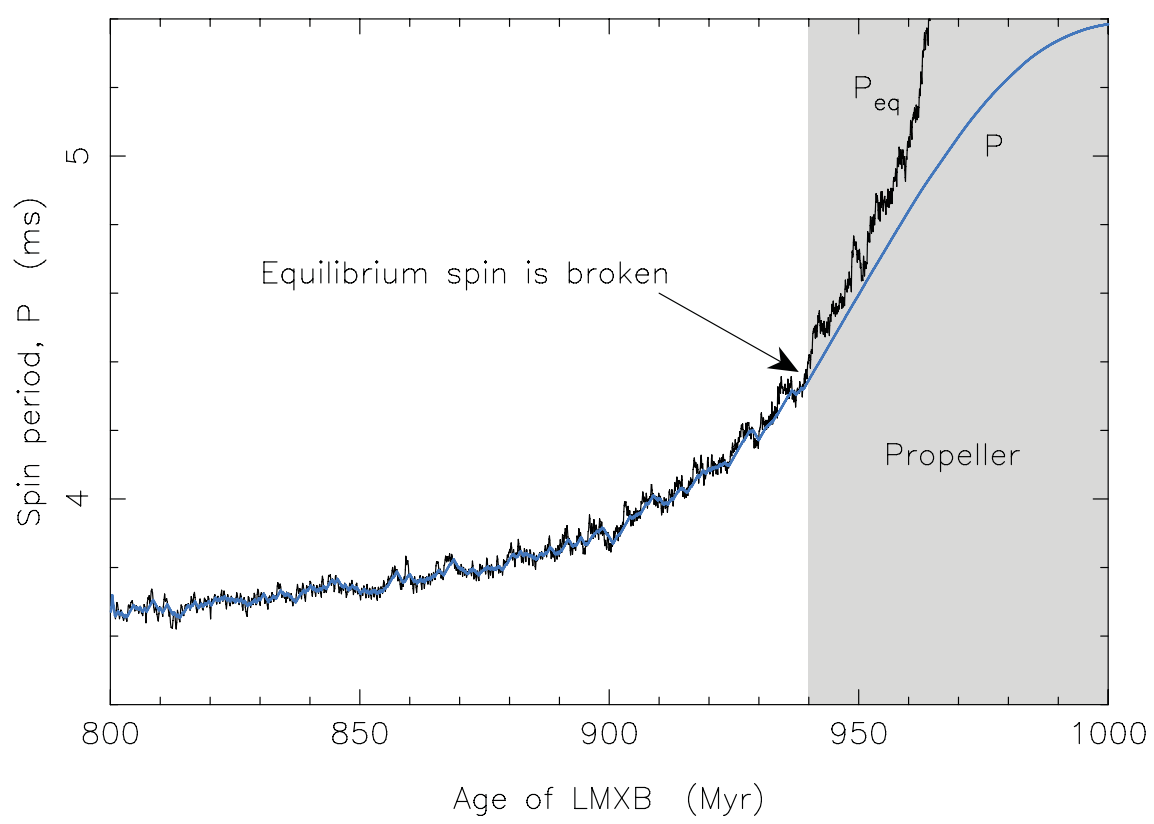

Figure 1. Transition from equilibrium spin to propeller phase. The black line is the equilibrium spin period of the neutron star, $P_{\text {eq }}$ (the oscillations reflect fluctuations in $\dot{M}(t)$ ) and the blue line is its actual spin period, $P$. At early stages of the Roche-lobe decoupling phase (RLDP) the neutron star spin is able to remain in equilibrium despite the outward moving magnetospheric boundary caused by decreasing ram pressure. However, at a certain point (indicated by the arrow), when the mass-transfer rate decreases rapidly, the torque can no longer transmit the deceleration fast enough for the neutron star to remain in equilibrium. (Tauris 2012.) 


\section{The spin-relaxation timescale and the duration of the RLDP}

To estimate the spin-relaxation timescale one can simply consider: $t_{\text {torque }}=J / N$ :

$$
t_{\text {torque }}=I\left(\frac{4 G^{2} M^{2}}{B^{8} R^{24} \dot{M}^{3}}\right)^{1 / 7} \frac{\omega_{c}}{\phi^{2} \xi} \simeq 50 \mathrm{Myr} B_{8}^{-8 / 7}\left(\frac{\dot{M}}{0.1 \dot{M}_{\mathrm{Edd}}}\right)^{-3 / 7}\left(\frac{M}{1.4 M_{\odot}}\right)^{17 / 7}
$$

In intermediate-mass X-ray binaries (IMXBs) the mass-transfer phase from a more massive companion is relatively short, causing the RLDP effect to be negligible. The reason for the major difference between the RLDP effect in LMXBs (often leading to the formation of radio MSPs with helium white dwarf companions) and IMXBs (primarily leading to mildly recycled pulsars with carbon-oxygen/oxygen-neon magnesium white dwarfs) is the time duration of the RLDP relative to the spin-relaxation timescale, i.e. the ratio of $t_{\mathrm{RLDP}} / t_{\text {torque }}$. In LMXBs, $t_{\mathrm{RLDP}} / t_{\text {torque }} \simeq 0.3$. However, in IMXBs this ratio is often smaller by a factor of 10 which causes the spin period to "freeze" at the original value of $P_{\text {eq }}$, see Tauris et al. (2012) for further details and examples of model calculations.

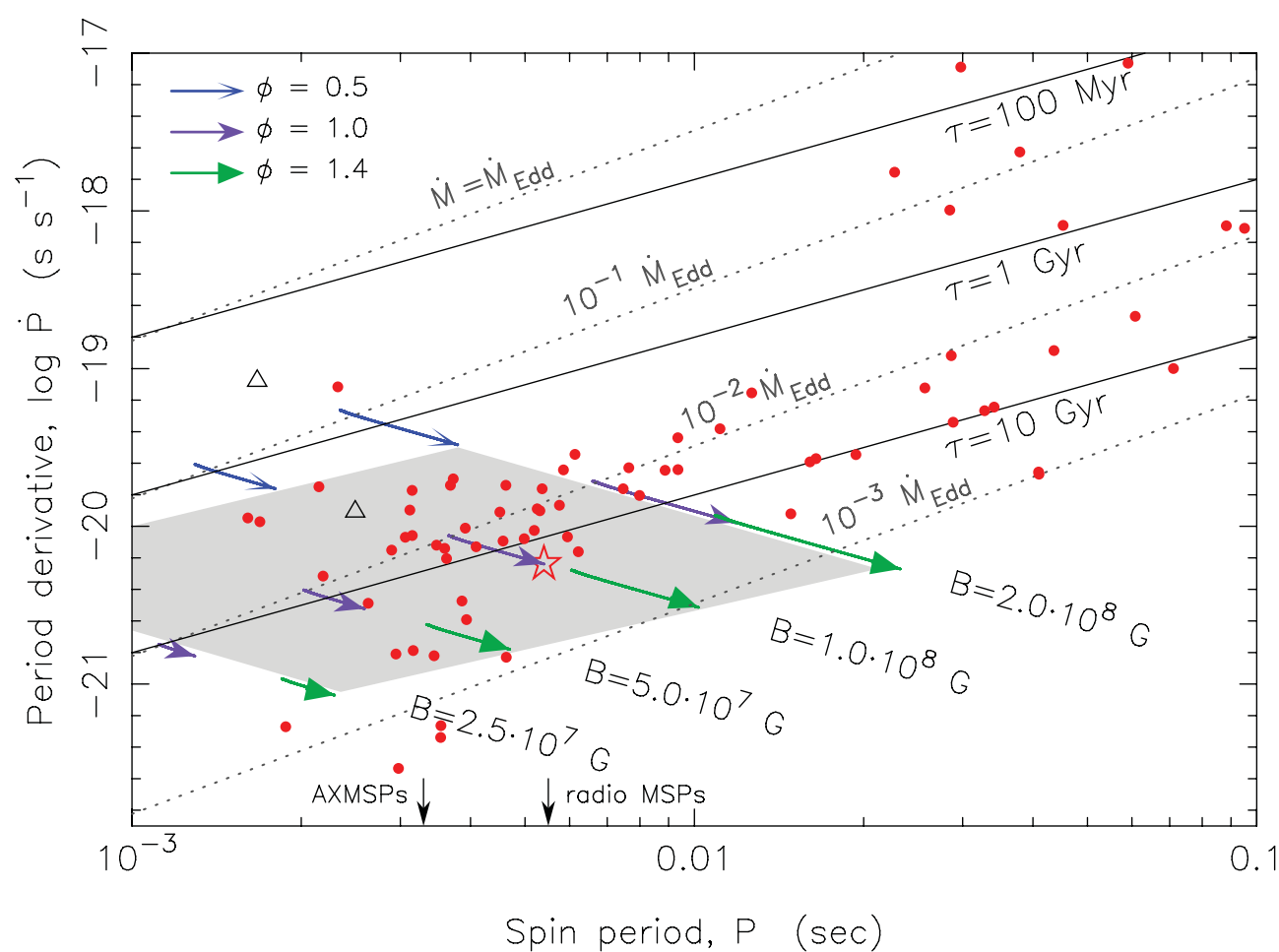

Figure 2. Evolutionary tracks during the Roche-lobe decoupling phase (RLDP). Computed tracks are shown as arrows in the $P \dot{P}$-diagram calculated by using different values of the neutron star B-field strength. The various types of arrows correspond to different values of the magnetospheric coupling parameter, $\phi$. The gray-shaded area indicates all possible birth locations of recycled MSPs calculated from one donor star model (marked by a star). The solid lines represent characteristic ages, $\tau$, and the dotted lines are spin-up lines calculated for a magnetic inclination angle, $\alpha=90^{\circ}$. The two triangles indicate approximate locations of the AXMSPs SWIFT J1756.92508 (upper) and SAX 1808.43658 (lower). Observed MSPs in the Galactic field are shown as dots [data taken from the ATNF Pulsar Catalogue, December 2011]. All the measured $\dot{P}$ values are corrected for the Shklovskii effect. The average spin periods of AXMSPs and radio MSPs are indicated with arrows at the bottom of the diagram. (Tauris 2012.) 


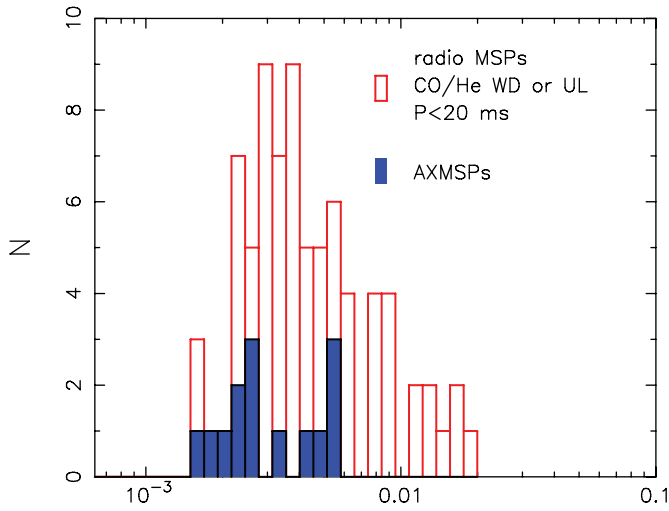

Spin period, $P(\mathrm{sec})$

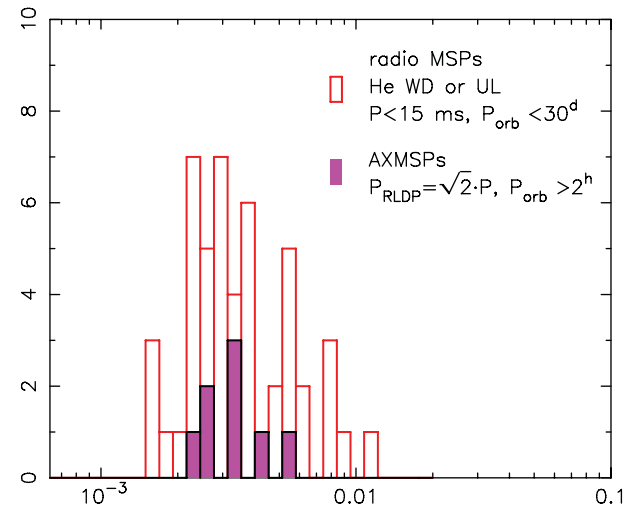

Spin period, $P(\mathrm{sec})$

Figure 3. Left panel: the observed distribution of all 78 Galactic radio MSPs (open red bins) and 14 AXMPS (solid blue bins). Right panel: 49 radio MSPs with He WD or ultra-light companions, $P<15 \mathrm{~ms}$ and $P_{\text {orb }}<30^{\mathrm{d}}$, together with the 8 AXMSPs, with $P_{\text {orb }}>2^{\mathrm{h}}$ which are likely to evolve into binary radio MSPs, corrected for the RLDP effect when these sources become radio MSPs by multiplying their spin periods by a factor of $\sqrt{2}$ (solid pink bins). The good correspondence between these two populations supports the RLDP-effect hypothesis.

\section{Spin distribution of AXMSPs and radio MSPs}

The RLDP effect discussed here can explain why the recycled radio MSPs (observed after the RLDP) are significantly slower rotators compared to the more rapidly spinning AXMSPs (observed before the RLDP), see Fig. 3. Only for radio MSPs with $B>10^{8} \mathrm{G}$ can the difference in spin periods be partly understood from regular magnetic dipole and plasma current spin-down over a radio MSP lifetime of several Gyr. When comparing radio MSPs and AXMSPs one should be aware of differences in their binary properties and observational biases, see SOM in Tauris (2012). Observationally, the RLDP effect can be verified if future surveys discover a significant number of AXMSPs and radio MSPs confined to an interval with similar orbital periods and companion star masses.

Although AXMSPs are believed to be progenitors of radio MSPs, all of the 14 observed AXMSPs have orbital periods less than one day whereas fully recycled radio MSPs are observed with orbital periods all the way up to a few hundred days. This is a puzzle. Some radio MSPs are born (recycled) with $B \simeq 1 \times 10^{7} \mathrm{G}$ and they would most likely not be able to channel the accreted matter sufficiently to become observable as AXMSPs.

Future modelling of the RLDP should ideally include irradiation effects, accretion disk instabilities and cyclic accretion (e.g. Spruit \& Taam 1993; D'Angelo \& Spruit 2010).

\section{References}

D'Angelo, C. R. \& Spruit, H. C. 2010, MNRAS, 406, 1208

Ghosh, P. \& Lamb, F. K. 1979, ApJ, 234, 296

Podsiadlowski, Ph., Rappaport, S. A., \& Pfahl, E. D. 2002, ApJ, 565, 1107

Rappaport, S. A., Fregeau, J. M., \& Spruit, H. C. 2004, ApJ, 606, 436

Ruderman, M., Shaham, J., \& Tavani, M. 1989, ApJ, 336, 507

Spruit, H. C. \& Taam, R. E. 1993, ApJ, 402, 593

Tauris, T. M. 2012, Science, 335, 561

Tauris, T. M. \& Savonije, G. J. 1999, A\&\&A, 350, 928

Tauris, T. M., Langer, N., \& Kramer, M. 2012, MNRAS, 425, 1601

Wang, Y.-M. 1997, ApJ, 475, L135 